\title{
Verzeichnis der Vortragenden
}

Kapitel 1

Prof. Dr. Gerd Gigerenzer

Direktor Harding Zentrum für Risikokompetenz, Max-Planck-Institut für Bildungsforschung

Lentzeallee 94, 14195 Berlin

E-Mail: sekgigerenzer@mpib-berlin.mpg.de

\section{Prof. Dr. Markus Knauff}

Justus-Liebig-Universität Gießen - Allgemeine Psychologie und Kognitionsforschung Otto-Behaghel-Straße 10, Haus F, 35394 Gießen E-Mail: markus.knauff@psychol.uni-giessen.de

\section{Kapitel 2}

Prof. Dr. Dr. h. c. Thomas C. Mettenleiter

Friedrich-Loeffler-Institut, Bundesforschungsinstitut für Tiergesundheit

Südufer 10, 17493 Greifswald - Insel Riems

E-Mail:ThomasC.Mettenleiter@fli.de

\section{Prof. Dr. Werner Solbach}

Universität zu Lübeck, Zentrum für Infektionsund Entzündungsforschung

Ratzeburger Allee 160, 23538 Lübeck

E-Mail: werner.solbach@uni-luebeck.de

\section{Kapitel 3}

\section{Dr. Andrea Ammon}

European Centre for Disease Prevention and Control (ECDC)

Granits Väg 8, 17165 Solna, SWEDEN

E-Mail: andrea.ammon@ecdc.europa.eu

\section{Prof. Dr. Ansgar W. Lohse}

Universitatsklinikum Hamburg-Eppendorf, I. Medizinische Klinik und Poliklinik Martinistraße 52, 20246 Hamburg

E-Mail: alohse@uke.de

\section{Kapitel 4}

Prof. Dr. Arne Traulsen

Max-Planck-Institut für Evolutionsbiologie August-Thienemann-Straße 2, 24306 Plön

E-Mail: traulsen@evolbio.mpg.de

\section{Prof. Dr. Dirk Langemann}

Technische Universität Braunschweig, Institut Computational Mathematics, AG PDE Universitätsplatz 2, 38106 Braunschweig E-Mail: d.langemann@tu-bs.de

\section{Kapitel 5}

Prof. Dr. Andreas Lange

Universität Hamburg - Fachbereich Volkswirtschaftslehre

Von-Melle-Park 5, 20146 Hamburg

E-Mail: Andreas.Lange@wiso.uni-hamburg.de

\section{Prof. Dr. Till Grüne-Yanoff}

KTH Royal Institute of Technology

Brinellvägen 32, 10044 Stockholm, SWEDEN

E-Mail: till.grune@abe.kth.se

\section{Kapitel 6}

\section{Oberbranddirektor i. R. Klaus Maurer}

Berufsfeuerwehr Hamburg

Westphalensweg 1, 20099 Hamburg

E-Mail: Poststelle@feuerwehr.hamburg.de

Prof. Dr. Edwin Kreuzer

Akademie der Wissenschaften in Hamburg Edmund-Siemers-Allee 1 (Ost), 20146 Hamburg E-Mail: praesident@awhamburg.de 
Kapitel 7

Polizeipräsident Ralf Martin Meyer

Bruno-Georges-Platz 1, 22297 Hamburg

E-Mail: pb@polizei.hamburg.de

Prof. Dr. Michael Fehling

Bucerius Law School

Jungiusstraße 6, 20355 Hamburg

E-Mail: michael.fehling@law-school.de

\section{Kapitel 8}

Prof. Dr. Till Grüne-Yanoff

KTH Royal Institute of Technology

Brinellvägen 32, 10044 Stockholm, SWEDEN

E-Mail: till.grune@abe.kth.se

Prof. Dr. Michael Brzoska

Institut für Friedensforschung

und Sicherheitspolitik

Beim Schlump 83, 20144 Hamburg

E-Mail: brzoska@ifsh.de 